\title{
Tc-99m hydroxymethylene diphosphonate scintigraphy, computed tomography, and magnetic resonance imaging of osteonecrosis in the mandible: Osteoradionecrosis versus medication- related osteonecrosis of the jaw
}

\author{
Ichiro Ogura $\mathbb{D}^{1, *}$, Yoshihiko Sasaki², Mikiko Sue ${ }^{2}$, Takaaki Oda ${ }^{2}$, Ayako Kameta $^{1}$, Kazuhide Hayama ${ }^{1}$ \\ ${ }^{1}$ Department of Oral and Maxillofacial Radiology, The Nippon Dental University, School of Life Dentistry at Niigata, Niigata, Japan \\ ${ }^{2}$ Radiology, The Nippon Dental University, Niigata Hospital, Niigata, Japan
}

\section{ABSTRACT}

Purpose: To present characteristic findings of Tc-99m hydroxymethylene diphosphonate (HMDP) scintigraphy, computed tomography (CT), and magnetic resonance (MR) imaging for osteonecrosis in the mandible, especially osteoradionecrosis (ORN) and medication-related osteonecrosis of the jaw (MRONJ).

Materials and Methods: Thirteen patients with MRONJ and 7 patients with ORN in the mandible underwent Tc-99m HMDP scintigraphy, CT, and MR imaging (T1-weighted images [T1WI], T2-weighted images [T2WI], short inversion time inversion recovery images [STIR]), diffusion-weighted images [DWI], and apparent diffusion coefficient [ADC] mapping). The associations of scintigraphy, CT, and MR imaging findings with MRONJ and ORN were analyzed using the chi-square test with the Pearson exact test.

Results: Thirteen patients with MRONJ and 7 patients with ORN in the mandible showed low signal intensity on T1WI and ADC mapping, high signal intensity on STIR and DWI, and increased uptake on scintigraphy. Periosteal bone proliferation on CT was observed in $69.2 \%$ of patients with MRONJ ( 9 of 13) versus $14.3 \%$ of patients with ORN(1 of 7) $(P=0.019)$.

Conclusion: This study presented characteristic imaging findings of MRONJ and ORN on scintigraphy, CT, and MR imaging. Our results suggest that CT can be effective for detecting MRONJ and ORN.(Imaging Sci Dent 2019; 49: 53-8)

KEY WORDS: Osteonecrosis; Tomography, X-Ray Computed; Radionuclide Imaging; Magnetic Resonance Imaging

\section{Introduction}

Bisphosphonates, which are inhibitors of osteoclastic bone resorption, ${ }^{1}$ are useful for the treatment of osteoporosis and bone metastases. ${ }^{2-6}$ However, they are also implicated in the onset of medication-related osteonecrosis of the jaw (MRONJ). ${ }^{1,7,8}$ Patients with MRONJ are often referred to a larger hospital for the evaluation of bisphosphonate-induced changes in the jaws, along with the differential diagnosis of other diseases of the jaws, such as osteoradionecro-

*This work was supported by JSPS KAKENHI Grant Number JP 18K09754.

Received October 30, 2018; Revised December 18, 2018; Accepted December 19, 2018 Correspondence to: Prof. Ichiro Ogura

Department of Oral and Maxillofacial Radiology, The Nippon Dental University, School of Life Dentistry at Niigata, 1-8 Hamaura-cho, Chuo-ku, Niigata, Niigata 9518580, Japan

Tel) 81-25-267-1500,E-mail) ogura@ngt.ndu.ac.jp sis (ORN) or osteomyelitis. ${ }^{9}$

ORN is a pernicious complication of radiotherapy for head and neck carcinomas. ${ }^{10}$ The most common cause of ORN is radiation arteritis, ${ }^{11-13}$ which leads to the onset of a hypocellular, hypovascular, and hypoxic environment. Jaws with MRONJ or ORN must be evaluated before any medical procedure is performed. ${ }^{9}$

Tc-99m hydroxymethylene diphosphonate (HMDP) scintigraphy is capable of demonstrating physiologic changes in bone, and it has been shown that scintigraphy is effective for detecting MRONJ. ${ }^{14}$ Furthermore, multiple imaging modalities, such as Tc-99m HMDP scintigraphy, computed tomography (CT), and magnetic resonance (MR) imaging, are useful for detecting MRONJ. ${ }^{8}$ However, to the best of our knowledge, the imaging features of MRONJ and ORN on scintigraphy, CT, and MR imaging have not been pre- 
sented in the literature. The aim of this study was to evaluate the Tc-99m HMDP scintigraphy, CT, and MR imaging findings of osteonecrosis in the mandible, especially ORN and MRONJ.

\section{Materials and Methods}

Thirteen patients with MRONJ and 7 patients with ORN in the mandible underwent Tc-99m HMDP scintigraphy, CT, and MR imaging at Radiology, The Nippon Dental
University Niigata Hospital from July 2013 to December 2017. Table 1 characteristics of the patients with ORN and MRONJ.

The images were acquired using a 16-MDCT apparatus (Aquilion TSX-101A; Canon Medical Systems, Otawara, Japan), a 1.5-T MR imaging system (EXCELART Vantage MRT-2003; Canon Medical Systems, Otawara, Japan), and a SNC-5100R scintigraphy apparatus (Shimadzu, Kyoto, Japan) with Tc-99m HMDP (Clear Bone Injection; Nihon Medi-Physics, Tokyo, Japan), following our institutional

Table 1. Characteristics of the patients with osteoradionecrosis (ORN) and medication-related osteonecrosis of the jaw (MRONJ)

\begin{tabular}{|c|c|c|c|}
\hline Number of patients & $\operatorname{ORN}(n=7)$ & $\operatorname{MRONJ}(\mathrm{n}=13)$ & $\operatorname{Total}(\mathrm{n}=20)$ \\
\hline \multicolumn{4}{|l|}{ Age (years) } \\
\hline Mean \pm standard deviation & $67.6 \pm 9.6$ & $75.6 \pm 10.0$ & $72.8 \pm 10.4$ \\
\hline Range & $52-84$ & $56-89$ & $52-89$ \\
\hline \multicolumn{4}{|l|}{ Sex } \\
\hline Male & 6 & 0 & 6 \\
\hline Female & 1 & 13 & 14 \\
\hline \multicolumn{4}{|l|}{ Primary disease } \\
\hline Tongue cancer & 3 & & 3 \\
\hline Oropharyngeal cancer & 3 & & 3 \\
\hline Floor of the mouth cancer & 1 & & 1 \\
\hline Osteoporosis & & 9 & 9 \\
\hline Osseous metastases of breast cancer & & 4 & 4 \\
\hline \multicolumn{4}{|l|}{ Medication } \\
\hline Alendronate & & 6 & 6 \\
\hline Risedronate & & 1 & 1 \\
\hline Minodronate & & 1 & 1 \\
\hline Zoledronate & & 1 & 1 \\
\hline Bevacizumab & & 2 & 2 \\
\hline Denosumab & & 1 & 1 \\
\hline Ibandronate & & 1 & 1 \\
\hline
\end{tabular}

Table 2. Comparison between osteoradionecrosis (ORN) and medication-related osteonecrosis of the jaw (MRONJ) with computed tomography (CT), magnetic resonance image (MRI) and bone scintigraphy

\begin{tabular}{lccc}
\hline \multicolumn{1}{c}{ Imaging features } & ORN $(\mathrm{n}=7)$ & MRONJ $(\mathrm{n}=13)$ & \\
\hline Bone scintigraphy & & & \\
Increased uptake & $7(100 \%)$ & & \\
CT images & & $13(100 \%)$ & \\
Osteolytic changes of the jaws & $6(85.7 \%)$ & $10(76.9 \%)$ & 0.639 \\
Sequestrum separation & $6(85.7 \%)$ & $9(69.2 \%)$ & 0.639 \\
Periosteal bone proliferation & $1(14.3 \%)$ & & 0.019 \\
MR images & & $13(100 \%)$ & $10(76.9 \%)$ \\
Low-signal intensity on T1WI & $7(100 \%)$ & $13(100 \%)$ & - \\
High-signal intensity on T2WI & $4(57.1 \%)$ & $8 / 8(100 \%)$ & - \\
High-signal intensity on STIR & $7(100 \%)$ & $8 / 8(100 \%)$ & - \\
High-signal intensity on DWI & $5 / 5(100 \%)$ & -357 \\
Low-signal intensity on ADC map & $5 / 5(100 \%)$ & - \\
\hline
\end{tabular}

T1WI: T1-weighted image, T2WI: T2-weighted image, STIR: short TI inversion recovery image, DWI: diffusion-weighted image, ADC: apparent diffusion coefficient 

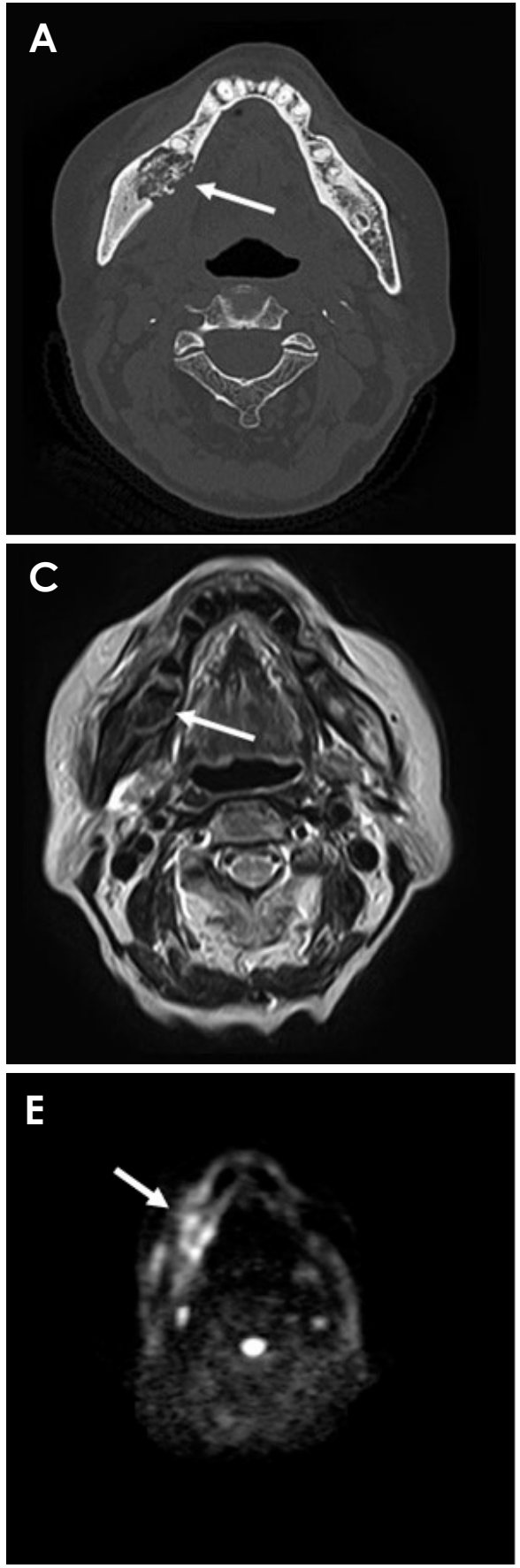

H

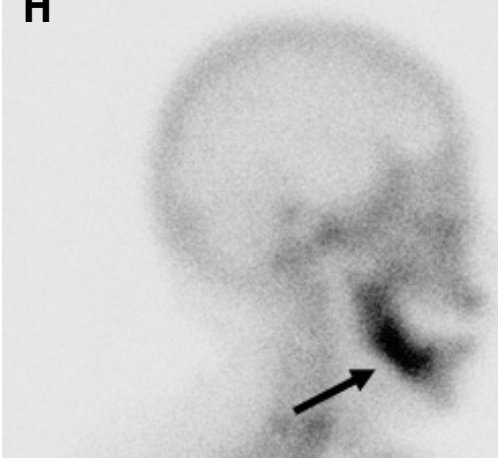

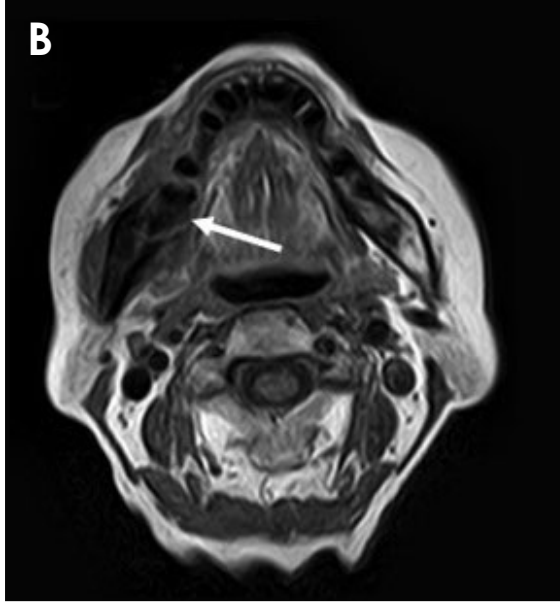

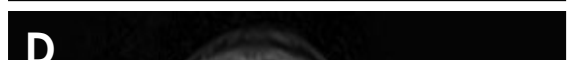

D

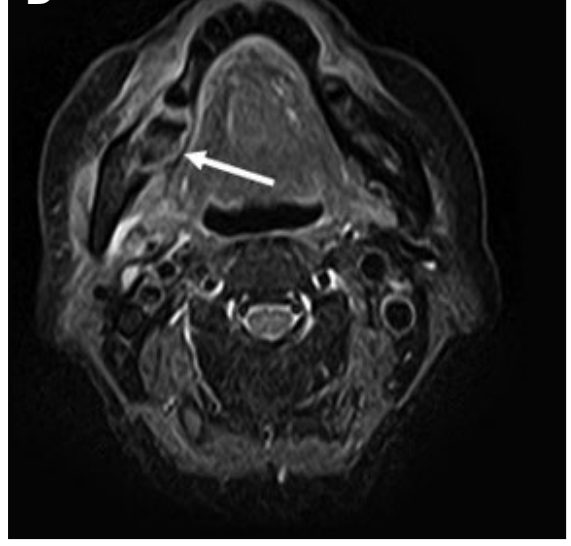

\section{$\mathbf{F}$}

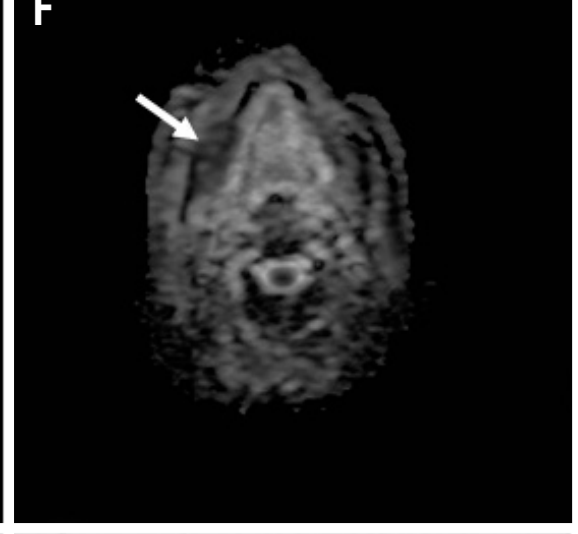

I

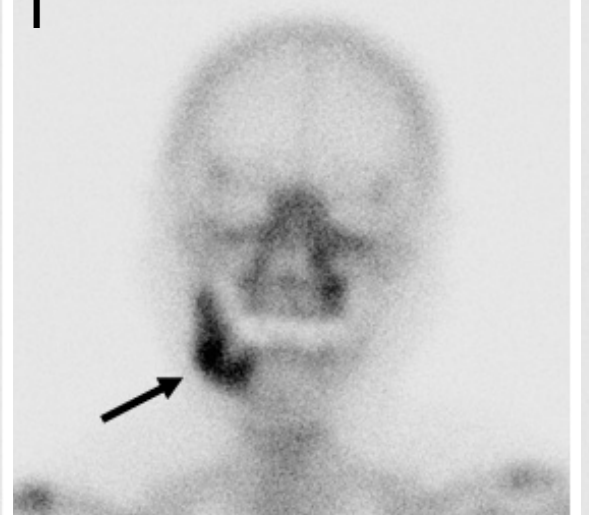

Fig. 1. Medication-related osteonecrosis of the right side of the mandible in an 86-yearold woman. A. Axial bone tissue algorithm computed tomography shows an osteolytic lesion with sequestrum separation and periosteal bone proliferation in the right mandible (arrow). B. On magnetic resonance imaging, an axial T1-weighted image shows heterogeneous, low-signal intensity (arrow). C. An axial T2-weighted image shows heterogeneous, low-signal intensity (arrow). D. An axial short TI inversion recovery image shows heterogeneous, high-signal intensity (arrow). E. An axial diffusion-weighted image shows heterogeneous, high-signal intensity (arrow). F. An axial apparent diffusion coefficient map shows heterogeneous, low-signal intensity (arrow). G. A maximum intensity projection (diffusion-weighted image) shows the lesion in an improved way (arrow). H-J. Bone scintigraphy shows increased uptake in the mandible (arrow).
G

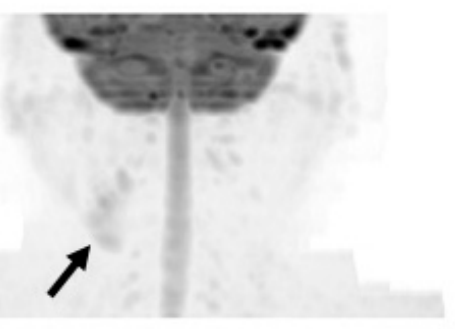



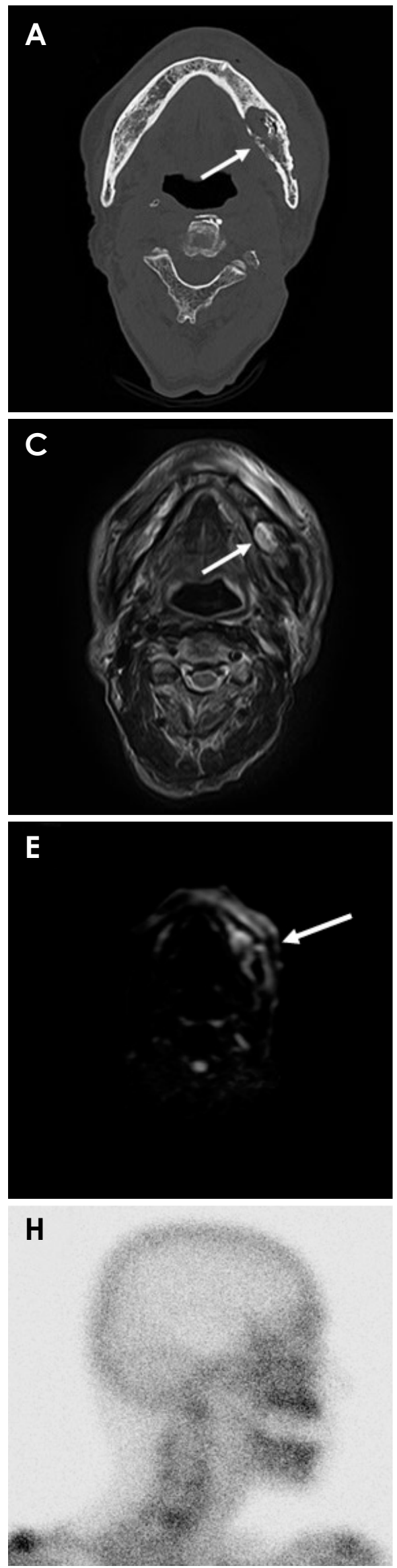
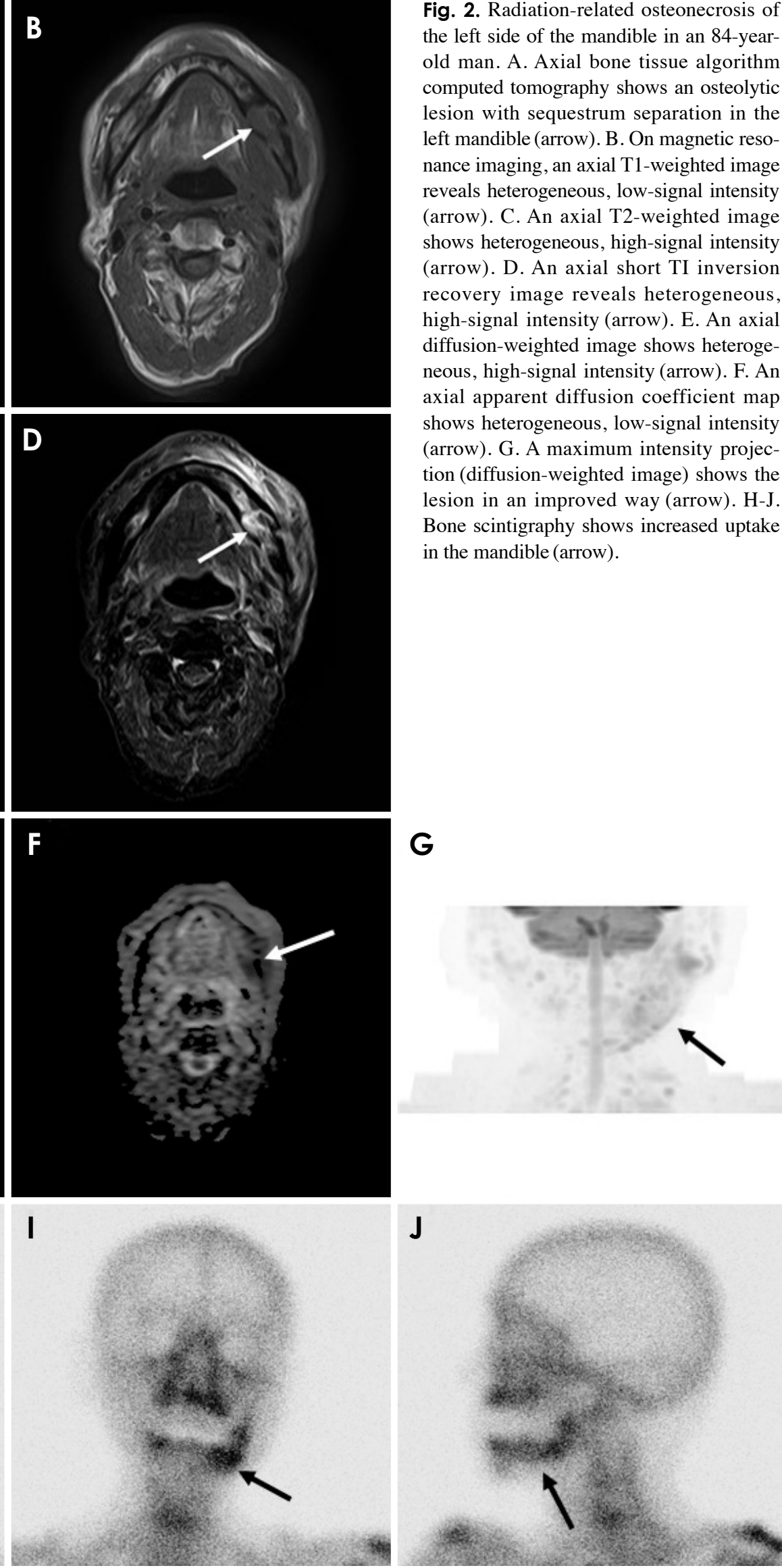

Fig. 2. Radiation-related osteonecrosis of the left side of the mandible in an 84-yearold man. A. Axial bone tissue algorithm computed tomography shows an osteolytic lesion with sequestrum separation in the left mandible (arrow). B. On magnetic resonance imaging, an axial T1-weighted image reveals heterogeneous, low-signal intensity (arrow). C. An axial T2-weighted image shows heterogeneous, high-signal intensity (arrow). D. An axial short TI inversion recovery image reveals heterogeneous, high-signal intensity (arrow). E. An axial diffusion-weighted image shows heterogeneous, high-signal intensity (arrow). F. An axial apparent diffusion coefficient map shows heterogeneous, low-signal intensity (arrow). G. A maximum intensity projection (diffusion-weighted image) shows the lesion in an improved way (arrow). H-J. Bone scintigraphy shows increased uptake in the mandible (arrow).

\section{G}

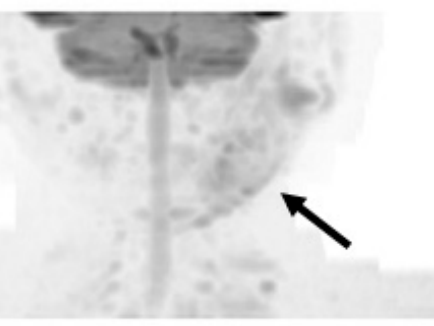


protocol. $^{8,14-16}$

The findings of Tc-99m HMDP scintigraphy, CT, and MR imaging of ORN and MRONJ were evaluated by 2 oral radiologists. Scintigraphy was used to analyze areas of increased uptake. CT was used to evaluate osteolytic changes of the jaws, sequestrum separation, and periosteal bone proliferation. In MR imaging, T1-weighted images (T1WI), T2-weighted images (T2WI), short inversion time inversion recovery images (STIR), diffusion-weighted images (DWI), and apparent diffusion coefficient (ADC) maps were obtained.

The associations of scintigraphy, CT, and MR imaging findings with MRONJ and ORN were analyzed using the chi-square test with the Pearson exact test. $P$ values $<0.05$ were considered to indicate statistical significance.

\section{Results}

The bone scintigraphy, CT, and MR imaging findings are compared between MRONJ and ORN in Table 2. Thirteen patients with MRONJ and 7 patients with ORN in the mandible showed low signal intensity on T1WI and ADC mapping, high signal intensity on STIR and DWI, and increased uptake on scintigraphy. Periosteal bone proliferation on CT was observed in $69.2 \%$ of patients with MRONJ (9 of 13) versus $14.3 \%$ of patients with ORN (1 of 7) $(P=0.019)$. Figures 1 and 2 show images of MRONJ and ORN, respectively.

\section{Discussion}

Imaging findings are unclear both in the early stages of ORN and when the disease is advanced. ${ }^{11-13}$ Although the radiological findings are nonspecific, they do appear to play a role in the management of MRONJ. ${ }^{17}$ In this study, we have presented the characteristic imaging findings of MRONJ and ORN on bone scintigraphy, CT, and MR imaging.

Tc-99m HMDP scintigraphy is an effective diagnostic tool for detecting bone changes, and has a higher sensitivity than that of radiography. ${ }^{14}$ Many authors have reported that scintigraphy showed increased uptake at sites affected by MRONJ. 5,17,18 In our study, all cases (MRONJ and ORN) showed positive findings on bone scintigraphy. Arce et al. ${ }^{3}$ reported that the presence of increased uptake in the bone on Tc-99m HMDP depends on osteoblastic activity and skeletal vascularity. We therefore suggest that scintigraphy shows areas of increased uptake at the sites affected by mandibular diseases.

Bisdas et al. ${ }^{9}$ reported that CT was useful for detecting predominantly osteolytic diseases and sclerotic lesions in the jaws, with or without periosteal bone proliferation. In our study periosteal bone proliferation on CT was observed in $69.2 \%$ of patients with MRONJ (9 of 13) versus $14.3 \%$ of patients with ORN ( 1 of 7$)(P=0.019)$. Therefore, we propose that periosteal bone proliferation is a distinctive characteristic of MRONJ on CT.

MR imaging is useful for the diagnosis of bone marrow disease, and necrosis is usually detected as areas of decreased signal on T1WI. ${ }^{13}$ Krishnan et al. ${ }^{2}$ reported that the earliest MR imaging finding of MRONJ was the loss of the normal T1 hyperintensity of fatty marrow in the mandible. In our study, all cases (ORN and MRONJ) showed low signal intensity on T1WI and high signal intensity on STIR. Driessen et al. ${ }^{19}$ reported that DWI demonstrated consistently high accuracy and high negative predictive value for head and neck cancer. Bonello et al. ${ }^{20}$ showed that DWI could provide information on microstructural tumor characteristics. Previous studies have reported that DWI and ADC maps were useful for the differential diagnosis of oral and maxillofacial lesions. ${ }^{15,16}$ In our study, all cases (ORN and MRONJ) showed high signal intensity on DWI, and low signal intensity on ADC maps. We therefore consider that DWI and ADC maps reflect the histopathological differences between malignant tumors and inflammatory diseases in the mandible.

Regarding the reasons underlying the different imaging characteristics of MRONJ and ORN, we propose that periosteal bone proliferation on CT in association with ORN is rare because $\mathrm{ORN}$ is caused by radiation arteritis. ${ }^{11-13}$

All cases of MRONJ and ORN in our study showed low signal intensity on T1WI and ADC maps, high signal intensity on STIR and DWI, and increased uptake on bone scintigraphy. We suggest that the increased uptake on bone scintigraphy may be correlated with MR imaging findings, especially those on DWI and ADC maps, although the sample was relatively small in this study.

In conclusion, this study presented the characteristic imaging findings of MRONJ and ORN on bone scintigraphy, $\mathrm{CT}$, and MR imaging. Our results suggest that CT can be effective for detecting MRONJ and ORN.

\section{References}

1. Taniguchi T, Ariji Y, Nozawa M, Naitoh M, Kuroiwa Y, Kurita $\mathrm{K}$, et al. Computed tomographic assessment of early changes of the mandible in bisphosphonate-treated patients. Oral Surg Oral Med Oral Pathol Oral Radiol 2016; 122: 362-72.

2. Krishnan A, Arslanoglu A, Yildirm N, Silbergleit R, Aygun N. Imaging findings of bisphosphonate-related osteonecrosis of the 
jaw with emphasis on early magnetic resonance imaging findings. J Comput Assist Tomogr 2009; 33: 298-304.

3. Arce K, Assael LA, Weissman JL, Markiewicz MR. Imaging findings in bisphosphonate-related osteonecrosis of jaws. J Oral Maxillofac Surg 2009; 67 (5 Suppl): 75-84.

4. Leite AF, Ogata Fdos S, de Melo NS, Figueiredo PT. Imaging findings of bisphosphonate-related osteonecrosis of the jaws: a critical review of the quantitative studies. Int J Dent 2014; 2014: 784348.

5. Paulo S, Abrantes AM, Laranjo M, Carvalho L, Serra A, Botelho MF, et al. Bisphosphonate-related osteonecrosis of the jaw: specificities. Oncol Rev 2014; 8: 254.

6. Koth VS, Figueiredo MA, Salum FG, Cherubini K. Bisphosphonate-related osteonecrosis of the jaw: from the sine qua non condition of bone exposure to a non-exposed BRONJ entity. Dentomaxillofac Radiol 2016; 45: 20160049.

7. Ruggiero SL. Diagnosis and staging of medication-related osteonecrosis of the jaw. Oral Maxillofac Surg Clin North Am 2015; 27: 479-87.

8. Ogura I, Sasaki Y, Kameta A, Sue M, Oda T. Characteristic multimodal imaging of medication-related osteonecrosis of the jaw: comparison between oral and parenteral routes of medication administration. Pol J Radiol 2017; 82: 551-60.

9. Bisdas S, Chambron Pinho N, Smolarz A, Sader R, Vogl TJ, Mack MG. Biphosphonate-induced osteonecrosis of the jaws: $\mathrm{CT}$ and MRI spectrum of findings in 32 patients. Clin Radiol 2008; 63: 71-7.

10. Chronopoulos A, Zarra T, Tröltzsch M, Mahaini S, Ehrenfeld M, Otto S. Osteoradionecrosis of the mandible: a ten year single-center retrospective study. J Craniomaxillofac Surg 2015; 43: 837-46.

11. Chronopoulos A, Zarra T, Ehrenfeld M, Otto S. Osteoradionecrosis of the jaws: definition, epidemiology, staging and clinical and radiological findings. A concise review. Int Dent J 2018; 68: 22-30.

12. van de Meent MM, Pichardo SE, Rodrigues MF, Verbist BM, van Merkesteyn JP. Radiographic characteristics of chronic dif- fuse sclerosing osteomyelitis/tendoperiostitis of the mandible: a comparison with chronic suppurative osteomyelitis and osteoradionecrosis. J Craniomaxillofac Surg 2018; 46: 1631-6.

13. Hatakeyama H, Fujima N, Tsuchiya K, Mizoguchi K, Mizumachi T, Sakashita T, et al. Osteoradionecrosis of the hyoid bone after intra-arterial chemoradiotherapy for oropharyngeal cancer: MR imaging findings. Cancer Imaging 2017; 17: 22.

14. Hayama K, Tsuchimochi M, Yamaguchi H, Oda T, Sue M, Kameta A, et al. Dynamic analysis of technetium-99m HMDP accumulation and its effect on regional bone metabolism and bone blood flow in bisphosphonate-related osteonecrosis of the jaw. Oral Radiol 2013; 29: 135-9.

15. Ogura I, Sasaki Y, Kameta A, Sue M, Oda T. Diffusion-weighted imaging in the oral and maxillofacial region: usefulness of apparent diffusion coefficient maps and maximum intensity projection for characterization of normal structures and lesions. Pol J Radiol 2017; 82: 571-7.

16. Oda T, Sue M, Sasaki Y, Ogura I. Diffusion-weighted magnetic resonance imaging in oral and maxillofacial lesions: preliminary study on diagnostic ability of apparent diffusion coefficient maps. Oral Radiol 2018; 34: 224-8.

17. Morag Y, Morag-Hezroni M, Jamadar DA, Ward BB, Jacobson JA, Zwetchkenbaum SR, et al. Bisphosphonate-related osteonecrosis of the jaw: a pictorial review. Radiographics 2009; 29 : 1971-84.

18. O'Ryan FS, Khoury S, Liao W, Han MM, Hui RL, Baer D, et al. Intravenous bisphosphonate-related osteonecrosis of the jaw: bone scintigraphy as an early indicator. J Oral Maxillofac Surg 2009; 67: 1363-72.

19. Driessen JP, van Kempen PM, van der Heijden GJ, Philippens ME, Pameijer FA, Stegeman I, et al. Diffusion-weighted imaging in head and neck squamous cell carcinomas: a systematic review. Head Neck 2015; 37: 440-8.

20. Bonello L, Preda L, Conte G, Giannitto C, Raimondi S, Ansarin M, et al. Squamous cell carcinoma of the oral cavity and oropharynx: what does the apparent diffusion coefficient tell us about its histology? Acta Radiol 2016; 57: 1344-51. 